\title{
Acquired Hepato Cerebral Degeneration - An Atypical Finding in MRI of Brain mimicking Bilateral Ischaemic Stroke
}

\author{
Abdul Kader Sheikh ${ }^{1}$, Mahjabin Islam ${ }^{2}$, Ahijit Chowdhury ${ }^{3}$, Ashraf Uddin Ahmed ${ }^{4}$, Utpal Dasgupta ${ }^{5}$

\begin{abstract}
:
Acquired Hepatocerebral Degeneration (AHD) produces a panel of neurological symptoms occurring in patients with chronic liver disease. These symptoms may mimic different neurological abnormalities e.g. abnormal movements, rigidity, parkinsonism, ataxia, neuropsychiatric and cognitive manifestations. ${ }^{[1]}$ Acquired Hepatocerebral Degeneration is usually encountered in patients with CLD due to accumulation of manganese \& other metabolic toxins in basal ganglia, following repeated episodes of liver failure ${ }^{[2]}$. The aim of this case report is to highlight the MRI finding of Acute Hepatocerebral Degeneration which often can be underappreciated owing to the possibility of diagnosing a relatively common condition that is Bilateral Ischemic stroke. [19].
\end{abstract}

Key words : Acquired Hepatocerebral Degeneration (AHD), Cirrhosis of Liver, Bilateral Ischemic Stroke.

\section{Introduction :}

Acquired Hepatocerebral Degeneration (AHD) is an infrequently diagnosed neurological syndrome characterized by a combination of neuropsychiatric, cognitive and extrapyramidal symptoms in patients with chronic liver disease, mostly in decompensated patients and patients with porto-systemic shunts. ${ }^{[1,2]}$ Though the pathophysiology of the disease is still not accurately postulated, a strong association of deposition of manganese in basal ganglia is traced as a basis of pathogenesis of Acquired Hepatocerebral Degeneration ${ }^{[3,4,5,6,7]}$. In elderly population, clinical features are often unclear and carry the chance of inaccurate diagnosis especially if MRI finding is not explained with expertise, as happened in our cases initially. For younger group of patients, Wilson's disease ${ }^{[\mathbf{1 0 ]}}$ may be another reason of misdiagnosis in the background of both hepatic and extrapyramidal symptoms. Here, we report two cases of Acquired Hepatocerebral Degeneration with clinical features, laboratory and radiological findings, precisely, the chance of under-appreciation of important radiological evidences prior to have a correct diagnosis.

1. Dr. Abdul Kader Shaikh, MBBS, FCPS (Medicine), MD (Neurology), Consultant Neurologist \& Internist, Square Hospitals LTD.

2. Dr. Mahjabin Islam, MBBS, Resident, Internal Medicine, Square hospitals LTD.

3. Dr. Ahijit Chowdhury, MBBS, Resident, ICU, Square hospitals LTD.

4. Dr. Ashraf Uddin Ahmed, MBBS, FCPS (Medicine), Specialist, Medicine, Square Hospitals LTD.

5. Dr. Utpal Dasgupta, MBBS, FCPS ( Medicine ), MD (Hepatology), Specialist, Medicine, Square Hospitals LTD.

\section{Corresponding Author :}

Dr. Mahjabin Islam (MBBS)

Resident Medical Officer

Square Hospitals LTD

18F Bir Uttam Kazi Nuruzzaman Road, Dhaka 1205

\section{Case Report-1 :}

A 59-year-old gentleman, driver by profession was admitted to the hospital with altered level of consciousness for 2 days. His wife mentioned that for the last 3 months, he had abnormal, unintentional hand and facial movements and difficulty in walking, change in speech and poor memory. On questioning, she mentioned that her husband was unable to remember the names of his relatives which he easily could even 1 year prior to this episode. For these symptoms, he was taken to a local doctor who had made a diagnosis of Idiopathic Parkinson's Disease and prescribed him with levodopa. He had no previous history of jaundice. On thorough physical examination, it revealed facial hypomimia, slurred speech, an ataxic gait, bilateral symmetrical resting tremor, and myoclonus, cogwheel rigidity in both wrists and flapping tremor of hands, with absence of limb spasticity and mute plantar response bilaterally. On mini mental state examination, he scored $19 / 30^{[7,8]}$ in detailing, found to have difficulty in memory recall, impaired calculation and complex word formation. Moderate ascites with splenomegaly was also evident. His biochemical parameters were altered with WBC count $3.34 \mathrm{k} / \mu \mathrm{L}$, Platelet count $78 \mathrm{k} / \mu \mathrm{L}$, Serum bilirubin-2.4 mg/dL, ALT-48 U, AST- $63 \mathrm{U}$, AFP- 4.73U, Ammonia-56, PT - $19.5 \mathrm{sec}$, INR 1.66, Albumin - $2.1 \mathrm{~g} / \mathrm{dl}$, Globulin - 4.3g/dl, A:G ratio 0.5. Serum copper and ceruloplasmin levels were normal. MRI of Brain was requested which revealed - On DWI, lesions at both centrum semiovales, capsuloganglionic region of both sides and cerebral \& cerebellar peduncles show increased signal intensity \& T2 flair images the same lesions show hyperintensity \& on T1WI these lesions show Hypointensities, but both globus pallidi were hyperintense. USG of Whole abdomen revealed small cirrhotic liver and moderate splenomegaly. Endoscopy of Upper Gastrointestinal tract revealed Grade III Oesophageal varices. After discussion, the complete diagnosis was Decompensated CLD with Acquired (Non-wilsonian) Hepatocerebral Degeneration. Patient was treated with gut sterilizing antibiotics, ammonia lowering agents, bowel relaxants and levodopa-Carbidopa for extrapyramidal Signs. The 
combination of multi modality treatment gradually improved his cognitive function, sleep pattern and consciousness level. Memory function and constructing abilities were yet to be assessed in the next follow up as it requires more time than predicted.

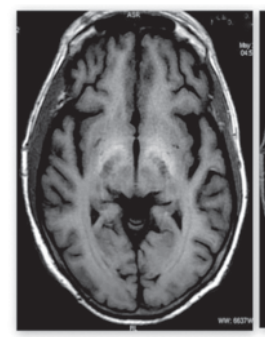

Image A

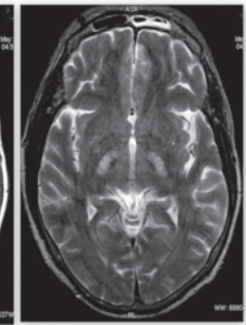

Image $B$

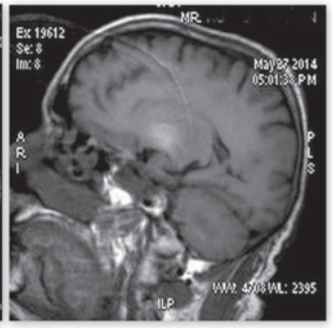

Image $C$
Fig 1 : Hypointensities in T1WI in ( Image A) Hyperintensities in T2-FLAIR -(Image B) - mimicking recent ischaemic stroke bilaterally involving Globus \& posterior limb of Internal Capsule .

\section{Case Report-2 :}

A 60-year-old school teacher was admitted to the hospital for gradual distension of abdomen and low urine output for 5 days. He had to get admitted twice before this admission in the same year. His son mentioned that the reasons for admission were poor sleep and altered consciousness level, latest 4 months prior to this admission. He recovered quite well after that episode. He had difficulty in remembering names of common objects. The most important point was elicited from the history given by the son that his father was upset as he could not remember the multiplication table to teach his grandson. 10 days prior to admission, the son had noticed altered sleep pattern, irrelevant talking, tremor in both hands and difficulty to initiate walk. After admission, physical examination revealed mild jaundice, distended veins in upper chest, marked ascites, splenomegaly and bilateral pedal edema. Neurological examination revealed masked face, dysarthric speech, nystagmus, bilateral hand tremor and mild rigidity in wrists and elbow and a typical Parkinson's gait. There was no spasticity, cranial nerve involvement or sensory impairment. Mini Mental State Examination also revealed a poor result. Routine blood tests were unremarkable with an exception in Platelet count $48 \mathrm{k} / \mu \mathrm{L}$, Bilirubin- $2.4 \mathrm{mg} / \mathrm{dl}$, ALT-78 IU, AST-105 IU, INR-1.76, Ammonia $-53 \mathrm{mcg} / \mathrm{dl}$, A:G ratio - 0.8 . Blood glucose, electrolytes, thyroid hormone, vitamin B12 level was normal. Endoscopy of Upper GastroIntestinal tract revealed Grade II Oesophageal varices. CT scan of abdomen demonstrated hepatic cirrhosis, splenomegaly and ascites as well as bilateral mild pleural effusion. MRI of brain showed hyperintensities of both globus pallidi on T1WI, Hypointensities in both centrum semiovales, splenium of corpus callosum, capsuloganglionic region of both sides and both cerebral peduncles, T2-flair images the same lesions showed hyperintensity, DWI lesions show increased signal intensity signifying restricted diffusion.

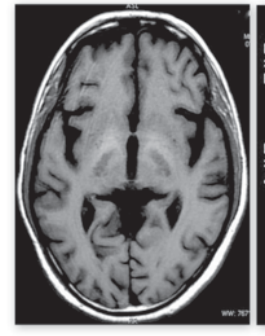

Image $A$
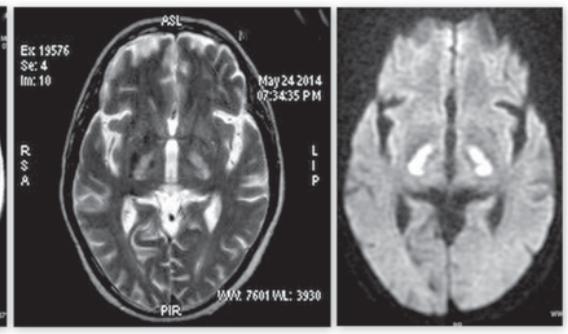

Image $B$
Image $C$
Fig 2 : Hypointensities in T1WI in (Image- A) Hyperintensities in T2-FLAIR-(Image- B) \& DW1 (Image C) \&-mimicking recent ischaemic stroke bilaterally involving Globus \& posterior limb of Internal Capsule .

\section{Discussion :}

Neurological dimension of a chronic liver disease patient is mostly straightforward as Hepatic Encephalopathy. Acquired Hepatocerebral Degeneration (AHD) has been diagnosed in a good proportion of patients with chronic liver disease who develop a wide entity of neurological symptomsparkinsonism, dementia, psychiatric symptoms may include apathy, lethargy, excessive somnolence, whereas extrapyramidal manifestations range from focal dystonia, postural tremor, akinesia, ataxia, myoclonus, choreoathetosis, ${ }^{[6,8]}$ also the usually overlooked entity which includes attention limitations, particularly visual-spatial components. ${ }^{[8,24]}$ This atypical chronic encephalopathy was first reported by van Woerkem in $1914^{[9]}$, but the term Acquired Hepatocerebral Degeneration was introduced in 1965 by Victor et al ${ }^{[10]}$ to distinguish the clinical manifestation in chronic liver disease from the genetic form, Wilson's disease. The exact pathophysiology of Acquired Hepatocerebral Degeneration (AHD) is under research. Review of different literatures made it evident that the disease is associated with a number of metabolic insults; manganese to be the major element causing the most detrimental effect, ${ }^{[6]}$ accumulates in the body fluids e.g. blood, cerebrospinal fluid due to defective hepatic clearance. ${ }^{[1,3,6,13]}$

The crucial part of diagnosis largely depends upon the accurate interpretation of MR images. Initial diagnoses, in patients who do not have overt features of liver disease, physicians often face the difficulty to comprehend the neurological symptoms in conjunction with hepatic features. As basal ganglia signal changes in MR imaging can be seen in a list of diseases for example osmotic myelinosis, Wilson's Disease, Hypoxic Ischaemic Encephalopathy, Wernicke's encephalopathy and Acquired hepatocerebral degeneration (AHD) ${ }^{[19]}$. In AHD, A characteristic MRI picture with bilaterally symmetric hyperintensities in globus pallidus and substantia nigra is seen in $71-92 \%$ of patients with cirrhosis [14]. Accumulation of manganese preferentially occurs in globus pallidus, substantia nigra pars reticulata, and striatum, as a result of impaired hepatic clearance of manganese in patients with cirrhosis ${ }^{[11,12,20]}$, hence rendering the characteristic image in MRI which may mimic Bilateral 
Ischaemic Stroke. Pallidal degeneration may occur due to toxic effects of manganese leading to loss of dopamine binding sites, often sparing the nigrostriatal system, in contrast to Parkinson disease, which preferentially damages dopaminergic neurons in the substantia nigra pars compacta. [19] Microscopically, a typical change, Alzheimer type II astrocytosis, as a result of alteration of protein andenzyme expression, deposition of cytoplasmic glycogen granules in basal ganglia may occur. ${ }^{[1,315,16]}$. MR imaging scans showed a hyperintense $\mathrm{T} 1$ signal in the basal ganglia. But it was also reported that some patients showed increased signal intensity in the in the ventral aspect of the midbrain and hypothalamus on T2-weighted image.

Burkhard et al. suggested hyperintensities, though mostly in the globus pallidus, can also be detected in the substantia nigra (some involving the ventral aspect of the midbrain, substantia innominata and hypothalamus) ${ }^{[12]}$ As accumulation of metabolic toxin in the brain takes time, patient with Acquired Hepatocerebral Degeneration usually develops overt features of CLD. But, like in our cases, it can be overlooked owing to the probability of the diagnosis of Idiopathic Parkinson's Disease in elderly population ${ }^{[25,26]}$ The MRI of brain with the typical changes in Basal Ganglia [4,12,21,22], altered Liver function test including Albumin: Globulin Ratio can bring up to the correct diagnosis of Acquired hepatocerebral degeneration (AHD). It is also necessary to exclude Wilson's Disease ${ }^{[17]}$ in younger age group which may have the similar neuro- hepatic symptoms and changes in brain which are indistinguishable in Neuroimaging, usually shows increased T2 signal in the basal ganglia, white matter, thalamus or brainstem.[12,26]. But, the elderly age group, normal copper level in blood \& absence of abnormal copper deposition in the cornea resulting in Kayser Fleischer ring can help to establish the differential. ${ }^{[17,18]}$

Elderly patients presented with dysarthria, ataxia or features of repeated episodes of cognitive decline, should undergo MRI scan and other liver function tests as often depending only on the symptoms of liver disease can yield underwhelming information and often the diagnosis of Acquired Hepatocerebral Degeneration can be delayed. MRI of brain with hyperintensities in basal ganglia is to be revised keenly. Reversal of $\mathrm{A} / \mathrm{G}$ ratio and low platelet count with higher level of ammonia should be regarded as important evidences, as seen in our patients. Treatment with a combination of Levodopa-Carbidopa showed good response which indicates this is a reversible phenomenon [6, 13, 27]. Surgical options have been explored, of which endovascular occlusion of a portosystemic shunt is found to be a temporarily effective option. Moreover, liver transplantation can be a curative treatment option in some cases ${ }^{[3,6,13]}$ where post transplantation follow up results were quite positive and regarded as a matter of hope for the treatment of Acquired Hepatocerebral Degeneration.

\section{Conclusion :}

In Middle aged \& elderly patients who present with extrapyramidal features, altered mental state \& poor memory function should always be looked for evidence of covert chronic liver disease. An MRI of brain can often be a matter of concern where it can mimic ischemic stroke bilaterally due to the pattern of manganese deposition in patients with Chronic Liver Disease. So correct diagnosis demands expertise and detailing to interpret clinical and radiological features of Acquired Hepatocerebral Degeneration.

\section{References :}

1. Jog MS, Lang AE. Chronic acquired hepatocerebral degeneration: case reports and new insights. Mov Disord 1995; 10:714722.

2. Ferrara J, Jankovic J. Acquired hepatocerebral degeneration. J Neurol 2009; 256:320332.

3. Layrargues GP. Movement dysfunction and hepatic encephalopathy. Metab Brain Dis 2001;16:2735

4. Butterworth RF, Spahr L, Fontaine S, Layrargues GP. Manganese toxicity, dopaminergic dysfunction and hepatic encephalopathy. Metab Brain Dis 1995; 10:259267.

5. Huang CC, Chu NS, Lu CS, Chen RS, Schulzer M, Calne DB. The natural history of Neurological manganism over 18 years. Parkinsonism Relat Disord 2007; 13 :143145.

6. Burkhard PR, D elavelle J, Du Pasquier R, Spahr L. Chronic parkinsonism associated with cirrhosis: A distinct subset of acquired hepatocerebral degeneration. A rch Neurol 2003; $60: 521528$

7. Stracciari A, Mattarozzi K, D'Alessandro R, Baldin E, Guarino M. Cognitive functioning in chronic acquired hepatocerebral degeneration. Metab Brain Dis 2008 ; 23: 155-160

8. Folstein MF, Folstein SE, McHugh PR. Mini Mental State: a practical method for grading the cognitive state of patients for clinician. J Psychiatr Res 1975; 12:189198.

9. Van Woerkem W. La cirrhose hepatique avec alterations dans les centres nerveux evoluant chez des sujets d'age moyen. Nouvelle Iconographie de la Salpetriere, Clinique des Maladies du Systeme Nerveux $1914 ; 7: 4145$.

10. Victor M, Adams RD, Cole M. The acquired (nonWilsonian) type of chronic hepatocerebral. Medicine 1965; 44: 345396.

11. Rodriguez R, Contreras A, De Villoria JG, Grandas F. Acquired hepatocerebral degeneration: Clinical characteristics and MRI findings. Eur J Neurol. 2010; $17: 1463-70$. [ PubMed ]

12. Hauser RA, Zesiewicz TA, Rosemurgy AS, Martinez C, Olanow $\mathrm{CW}$. Manganese Intoxication and chronic liver failure. Ann Neurol $1994 ; 36: 871875$.

13. Condat B, Dusoleil A, Bernardeau M, Roche A, Pelletier G, Buffet C. Chronic acquiredhepatocerebral degeneration: the role of manganese and treatment by endovascular occlusion of a portosystemic shunt. Gastroenterol Clin Biol 1999; 23: 268270

14. P R. Delavelle J, Du Pasquier R, Spahr L. Chronic Parkinsonism associated with cirrhosis: A distinct subset of acquired hepatocerebral degeneration. Arch Neurol. 2003; 60:521-8. [ PubMed ]

15. Finlayson $\mathrm{MH}, \mathrm{S}$ uperville B. Distribution of cerebral lesions in acquired hepatocerebral degeneration. Brain 1981; 104 (Pt 1): 7995 
16. Soffer D, Sherman Y, Tur Kaspa R, Eid A. Acquired hepatocerebraldegeneration in a liver transplant recipient. Acta Neuropathol 1995; 90 : 107111

17. Levy VG, C ameron E, Ollat H, Opolon P, Darnis F, ContaminF. Chronic hepatic encephalopathies.

Acquired cerebral degeneration not due to Wilson's disease . S em Hop 1983; 59:13691373

18. Spencer DC, F orno LS. February 2000: Dementia with motor dysfunction in a patient with liver disease. Brain Pathol 2000; 10:315316

19. B. Smita, V. Abdul Gafoor, K . Saifudheen, and J ames Jose. Acute stroke like presentation of acquired hepatocerebral degeneration Ann Indian Acad Neurol. 2014 AprJun; 17(2): 204-206.

20. Spahr LButterworth RFontaine $\mathrm{S}$ et al Increased blood manganese in cirrhotic patients: relationship to pallidal magnetic signal hyperintensity and neurological symptoms. Hepatology. 1996; 24: 11161120.

21. Krieger D Krieger S Jansen O Gass P Theilmann L Lichtnecker H Manganese and chronic hepatic encephalopathy. Lancet. 1995; 346 :270274.
22. Saito Hejima A Liver dysfunction and probable manganese accumulation in the brainstem and basal ganglia. J Neurol Neurosurg Psychiatry.

$1995 ; 58: 760761$.

23. Waggoner R Malamud N Wilson's disease in the light of cerebral changes following ordinary acquired liver disorders. J Nerv Ment Dis. 1942; 96:410435

24. Pinarbasi B, Kaymakoglu S, Matur Z, et al. Are acquired hepatocerebral degeneration and hepatic myelopathy reversible? J Clin Gastroenterol 2009; 43: 1761-1781.

25. Chen WX, Wang P, Yan SX, Li YM, Yu CH, Jiang LL. Acquired hepatocerebral degeneration: a case report. World J Gastroenterol 2005; 11:764-766.

26. Park HK, Kim SM, Choi CG, Lee MC, Chung SJ. Effect of trientine on manganese intoxication in a patient with acquired hepatocerebral degeneration. Mov Disord 2008;23:768-770.

27. Ueki Y, Isozaki E, Miyazaki Y, Koide R, Shimizu T, Yagi K, Hirai S. Clinical and neuroradiological improvement in chronic acquired hepatocerebral degeneration after branched-chain amino acid therapy. Acta Neurol Scand 2002; 106: 113-116 